\title{
Experimental and Theoretical Study on Influence of Different Charging Structures on Blasting Vibration Energy
}

\author{
Wenbin Gu, Zhenxiong Wang, Jianghai Chen, Jianqing Liu, and Ming Lu \\ College of Field Engineering, PLA University of Science and Technology, Nanjing, Jiangsu 210007, China \\ Correspondence should be addressed to Zhenxiong Wang; wangzhenxiong70310@126.com
}

Received 20 April 2015; Revised 1 July 2015; Accepted 2 July 2015

Academic Editor: Mario Terzo

Copyright ( 2015 Wenbin Gu et al. This is an open access article distributed under the Creative Commons Attribution License, which permits unrestricted use, distribution, and reproduction in any medium, provided the original work is properly cited.

\begin{abstract}
As an important parameter in blasting design, charging structure directly influences blasting effect. Due to complex conditions of this blasting and excavating engineering in Jiangsu, China, the authors carried out comparative researches with coupling structure, air-decoupling structure, and water-decoupling structure. After collecting, comparing, and analyzing produced signals on blasting vibration, the authors summarized that when proportional distances are the same, water-decoupling structure can reduce instantaneous energy of blasting vibration more effectively with more average rock fragmentation and less harm of dust. From the perspective of impedance matching, the present paper analyzed influence of charging structure on blasting vibration energy, demonstrating that impedance matching relationship between explosive and rock changes because of different charging structures. Through deducing relationship equation that meets the impedance matching of explosive and rock under different charging structures, the research concludes that when blasting rocks with high impedance, explosive with high impedance can better transmits blasting energy. Besides, when employing decoupling charging, there exists a reasonable decoupling coefficient helping realize impedance matching of explosive and rock.
\end{abstract}

\section{Introduction}

In rock blasting of engineering, energy that can be effectively used through explosion approximately accounts for $60 \sim 70$ percent of the total explosive energy. The rest may be used in exploding surrounding medium or produce harmful effects, such as blasting vibration, blasting shock wave, blasting flying blasting, blasting soot, blasting noise, and blasting harmful gas $[1,2]$; thus, it is of great significance to improve effective use of blasting vibration energy. According to damage characteristics of rock blasting, with explosion as the center, there are crushed zone, fracture zone, and vibration zone [3], from the center to distant area, and how to apply more explosive energy to fracture zone is key to engineering on rock blasting and excavating. Parameters affecting geotechnical blasting results are mainly on characteristics of rock and characteristics of explosive and blasting parameters and specific parameters are over 20 categories. When blasting environment and explosive are determined, charging structure plays a pivotal role in influencing blasting energy transmission. With decoupling charging, decoupling way between blasting hole and blasting charging, decoupling coefficient, compressible degree of decoupling medium (air or water), and initiating position of charging will largely affect transmitting process of blasting energy. Aimed at investigating charging structure's influence on blasting energy and effect, scholars both at home and abroad have carried out numerous researches on experiment and numerical simulation. Day examined distribution of explosive's pressure on rock hole wall of air-decoupling, water-decoupling and coupling charging structures with the same distance explosive conditions [4]. Zong and Meng analyzed effects of different charging structures of blasting hole on blasting energy and concluded that decoupling charging structure is beneficial under certain conditions [5]. Chen et al. conducted experiments on charging structure's influence on blasting seismic effects and concluded attenuation law of particle vibration velocity and vibration frequency [6]. Wang and Li carried out comparative numerical calculation on different radial coupling coefficient of water-decoupling charging structure in infinite concrete medium and then comprehensively analyzed relationships between damage zone distribution and pore wall pressure and acceleration as 


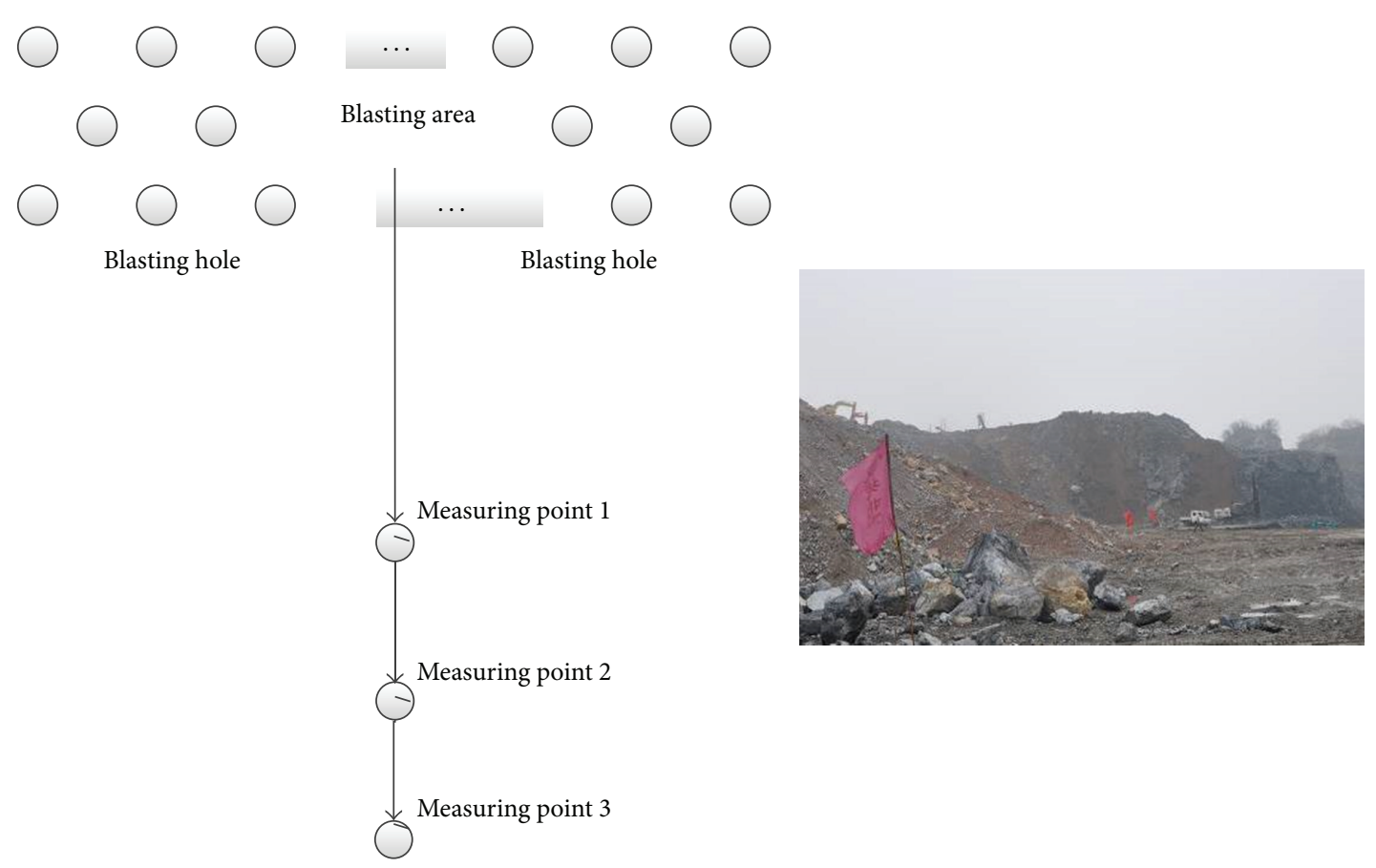

FIGURE 1: Schematic diagram and site layout of measuring points and blasting region.

well as velocity and radial decoupling coefficient [7]. Pape et al. carried out finite-element analysis methods, studied the explosion phenomena and effects of explosions on structures systematically, and predicted the harmful effects of blasting with different criterions $[8,9]$. By evaluating charging structure's influence on blasting effect and blasting vibration of near area, a large number of engineering practices, tests, and numerical simulations on charging structure indicate that decoupling charging structure can enhance utilization rate of explosive energy and improve blasting effect. However, in practical engineering, different construction environment requires different charging structures, yet there are not enough studies about analysis of energy transmission and blasting effects of different charging structures as well as carried energy of blasting vibration of far region of blasting. Hence, the present paper intends to explore relevant factors to provide an insightful reference for further investigations.

During rock excavation of engineering scene in Jiangsu province, researchers adopted rock drilling blasting method, which is widely applied in geotechnical engineering. Because of complexity of test scene and condition, the test accordingly selected different blasting charging structures. Through collecting blasting vibration signals, vibration speed was obtained, and so was instantaneous explosion vibration energy, and it was concluded that different charging structures' attenuation law of blasting vibration is different [10]. After explosion, observation on rock fragmentation of blasting area revealed that distribution of rock fragmentation produced by different blasting charging structures is obviously different. Based on the test, this research theoretically analyzed energy transmission of different charging structures from the perspective of impedance matching. The present study was designed to deduce the law of different charging structures' influence on blasting vibration energy of far region of blasting and provide reference for controlling side-effects of blasting vibration and designing parameters of charging structure.

\section{Experimental Research}

2.1. Set of Tests. To determine the influence of different charging structures on blasting vibration energy, the present paper took a blasting engineering in Jiangsu, China, as the example, carrying out experimental researches on the transmission of seismic wave of coupling charging, water-decoupling charging, and air-decoupling charging structures. Through test and analysis on vibration velocity of three charging structures in far region of blasting, blasting vibration energy was calculated.

Blasting network employed in experiment was multiple row hole blasting with differential time of $25 \mathrm{~ms}$ and two holes were blasted together; consequently, dosage of the two holes should be counted together to get the single detonation quantity in fitting calculating formula. The hole diameter was $0.09 \mathrm{~m}$, and, in experiments of decoupling charging, charging diameter was $0.07 \mathrm{~m}$, hole depth was $10 \mathrm{~m}$, and charging length was $7 \mathrm{~m}$. Schematic diagram as well as site layout of blasting region and measuring points are clarified in Figure 1.

Tests monitored vibration velocity with blasting vibration tester TC-4850 produced by Zhongke Measurement and Control Company. The tester has three parallel channels for monitoring vibration velocity in three directions; sampling rate is $1 \sim 50 \mathrm{KHz}$, with frequency response range $0 \sim 10 \mathrm{KHz}$. TC-4850 is widely used in engineering and its stability as well as accuracy can meet test requirements. In order to better gather blasting vibration signals, the test fixed collecting part 
TABLE 1: Peak vibration velocity of different charging structures at measuring points $(\mathrm{cm} / \mathrm{s})$.

\begin{tabular}{|c|c|c|c|c|c|c|}
\hline Charging structure & Proportional distance $r$ & $v_{\max }^{x}$ & $v_{\max }^{y}$ & $v_{\max }^{z}$ & $v_{\text {sum }}$ & $v_{\text {formula }}$ \\
\hline \multirow{3}{*}{ Coupling charging } & 4.281 & 14.361 & 11.769 & 19.666 & 20.180 & $6.614 \sim 25.986$ \\
\hline & 8.561 & 4.136 & 0.003 & 7.652 & 7.936 & $2.339 \sim 10.554$ \\
\hline & 12.842 & 3.143 & 3.244 & 3.430 & 4.748 & $1.273 \sim 6.230$ \\
\hline \multirow{6}{*}{ Air-decoupling charging } & 13.922 & 1.305 & 2.155 & 1.814 & 2.561 & $0.962 \sim 4.888$ \\
\hline & 14.228 & 1.753 & 1.003 & 1.108 & 2.481 & $0.932 \sim 4.754$ \\
\hline & 18.573 & 0.667 & 0.003 & 1.924 & 1.991 & $0.625 \sim 3.363$ \\
\hline & 21.341 & 1.360 & 0.757 & 0.888 & 1.792 & $0.507 \sim 2.806$ \\
\hline & 23.208 & 1.210 & 1.242 & 0.962 & 1.671 & $0.447 \sim 2.516$ \\
\hline & 30.826 & 1.096 & 0.677 & 0.767 & 1.237 & $0.292 \sim 1.740$ \\
\hline \multirow{3}{*}{ Water-decoupling charging } & 12.747 & 1.103 & 1.952 & 2.483 & 2.654 & $1.099 \sim 5.484$ \\
\hline & 16.995 & 0.574 & 1.320 & 0.943 & 1.618 & $0.714 \sim 3.773$ \\
\hline & 25.493 & 0.534 & 0.003 & 1.119 & 1.170 & $0.388 \sim 2.227$ \\
\hline
\end{tabular}
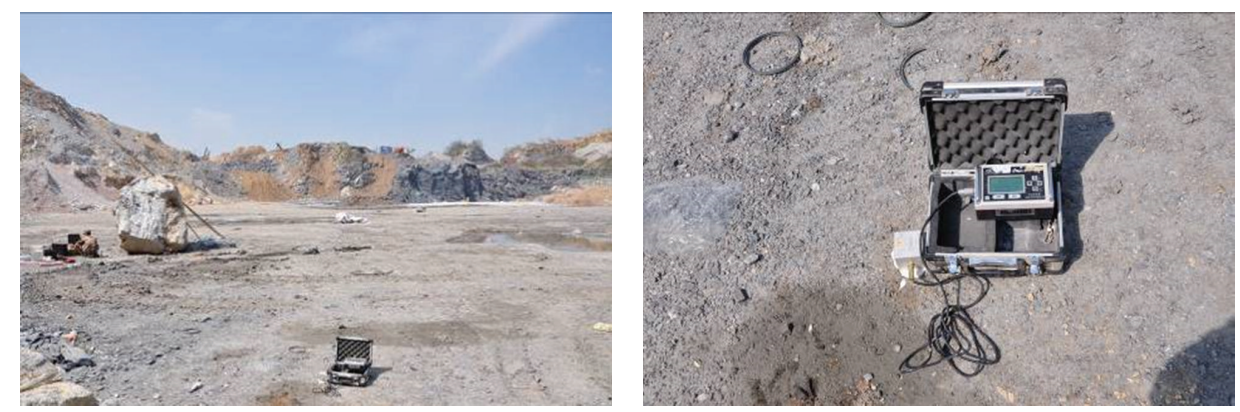

FIGURE 2: Site layout of blasting vibration tester.

of TC-4850 on the ground with gypsum and the site set is apparent in Figure 2.

2.2. Test Results. Prediction of blasting vibration is usually judged by peak vibration velocity of the particle, and the most commonly used one is Steve Sadove formula, where relationship between peak vibration velocity and proportional distance is [11]

$$
v=k r^{-\alpha} \text {. }
$$

Here, $r$ is for proportional distance, and its calculating formula is $r=R / \sqrt[3]{Q}$, where $R$ is the distance from blasting hole with $\mathrm{m}$ as the unit; $k$ and $\alpha$ are coefficients associated with explosive conditions and rock properties. $Q$ is for the total dosage of one time with $\mathrm{kg}$ as the unit. Rock in test area is limestone that belongs to middle-hard rock, according to whose characteristics, $k$ 's value range in the formula is $50 \sim 150$, and $\alpha$ 's value range is $1.3 \sim 1.5$ [12].

Figure 3 is time-history curve of vibration velocity and power spectrum of different charging structures. The main frequency of blasting vibration is about $0 \sim 200 \mathrm{~Hz}$, and, to reduce high frequency's influence on blasting vibration signals, those high frequency components are processed by software in data analysis to get the table of peak vibration velocity, as is demonstrated in Table 1 . The superscript stands for direction. In Table $1, v_{\text {sum }}$ 's are in the data range deduced and calculated from experience formula (1), so the test data are reliable. From those data, it can be seen that particle velocity of measuring points in three directions varies numerically, and peak value of the single component will also vary under different blasting environments, time, and frequency. So it is reasonable to choose square root from the square sum of three portions' peak speed as the particle's peak vibration velocity. The value of peak vibration velocity of the particle is [13]

$$
\left|v_{\text {sum }}\right|=\sqrt{\left(v_{x}\right)^{2}+\left(v_{y}\right)^{2}+\left(v_{z}\right)^{2}} \text {. }
$$

Therefore, to better predict blasting vibration, it may be most reasonable to evaluate it with module of the total vibration velocity vector (like formula (2)) [14]. With analysis of test data through least square method [15], it is indicated that, for different charging structures, values of $k$ and $\alpha$ in vibration velocity formula are demonstrated in Table 2. And Figure 4 displays data fitting curve of different charging structures.

Table 2 clearly indicates that there exist huge distinctions among coefficients in vibration predicting formula fitted for different charging structures. To better compare and analyze three charging structures' influence on blasting vibration, $\alpha$ can be supposed as 1.3; then the analysis can be realized with comparison of $k$ 's values. Through calculating and data fitting, when $\alpha=1.3$ and proportional distance $r$ are 

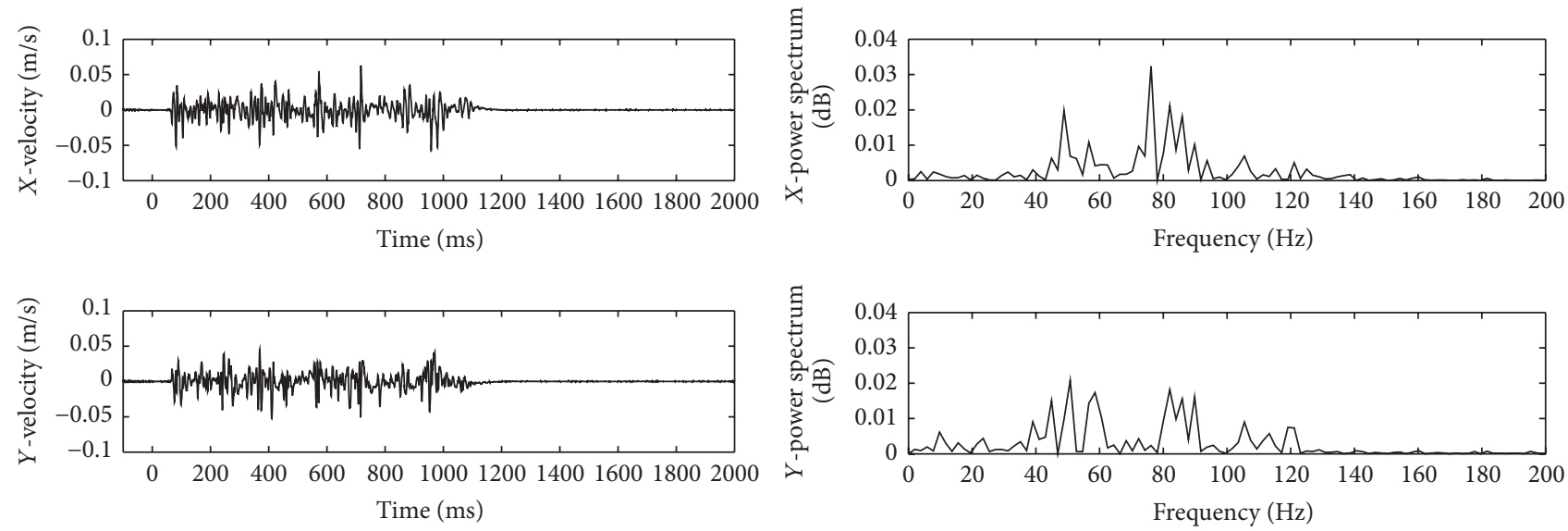

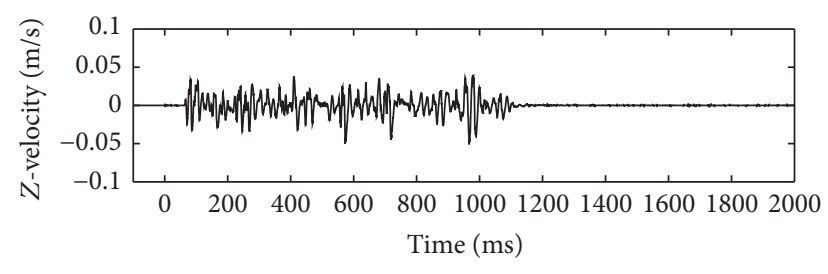

(A) Time-history curve of vibration velocity

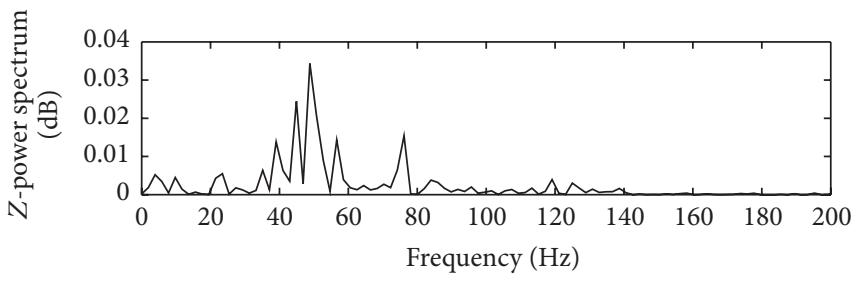

(B) The power spectrum

(a) Coupling charging structure $r=12.842$
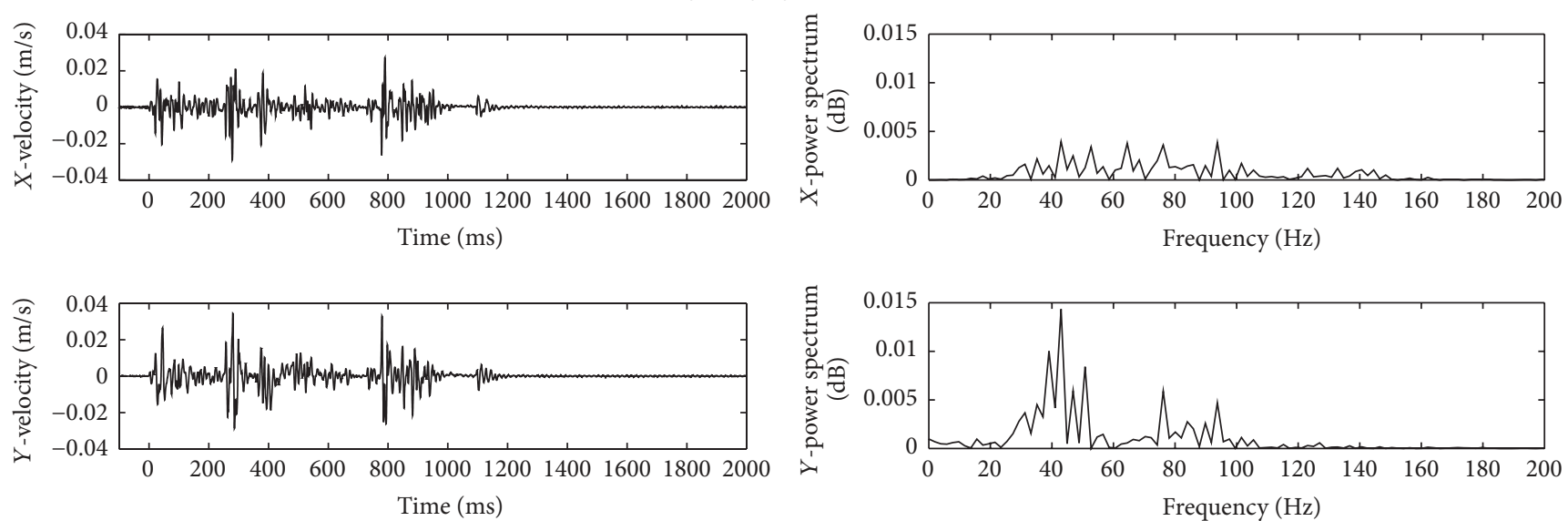

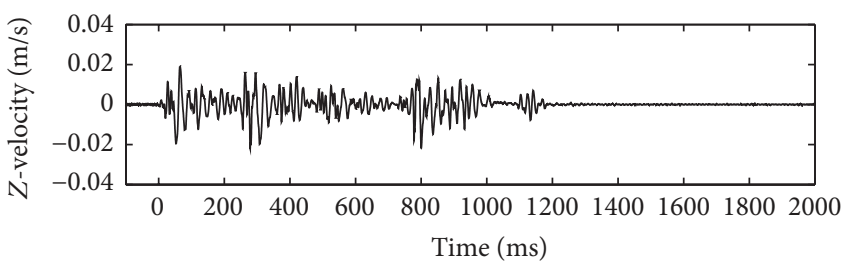

(A) Time-history curve of vibration velocity

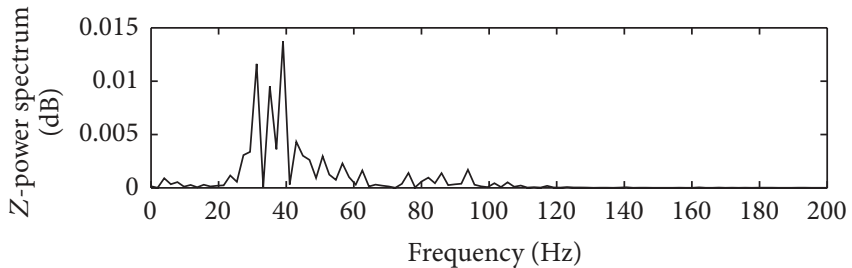

(B) The power spectrum

(b) Air-decoupling charging structure $r=13.922$
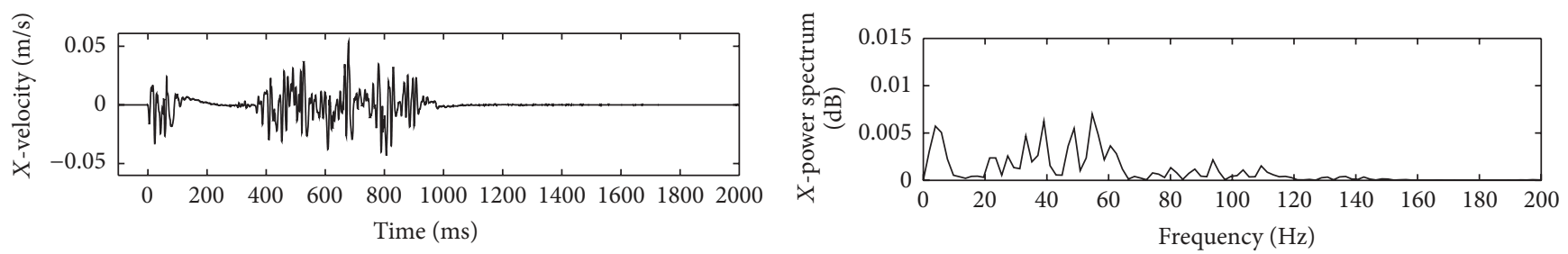

FIgUre 3: Continued. 

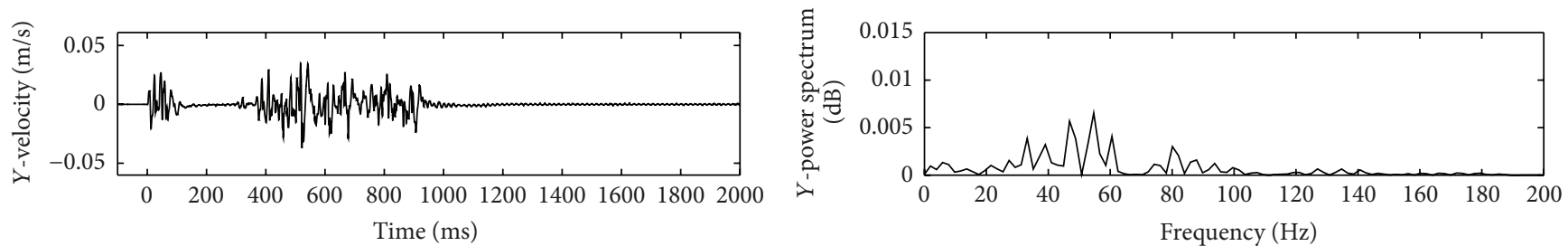

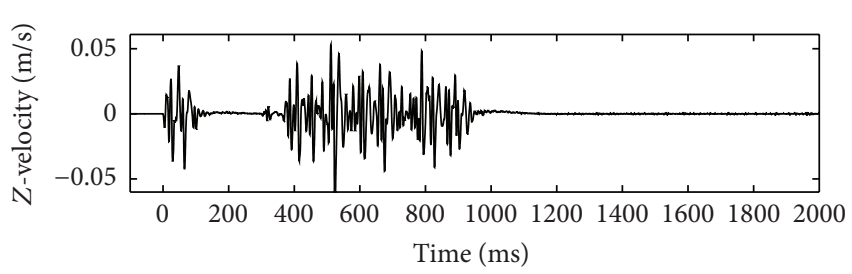

(A) Time-history curve of vibration velocity

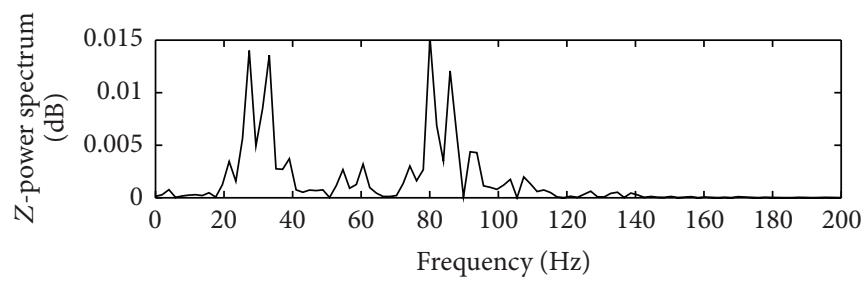

(B) The power spectrum

(c) Water-decoupling charging structure $r=12.747$

FiguRE 3: Schedule chart of different charging structures' vibration velocity and power spectrum in three directions.

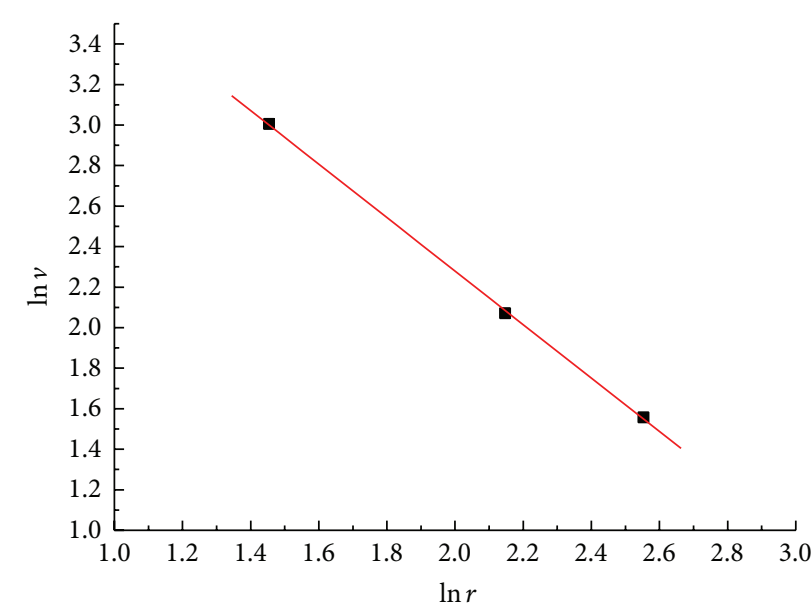

(a) Coupling charging structure

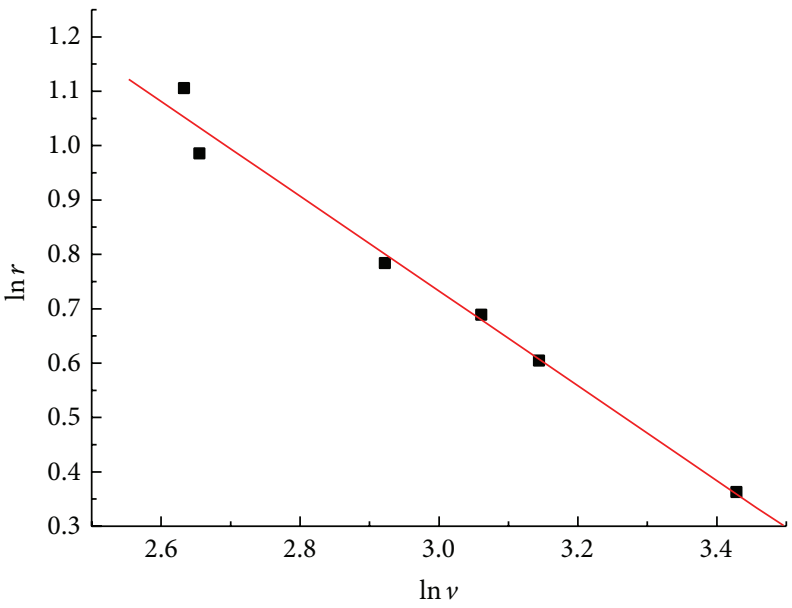

(b) Air-decoupling charging structure

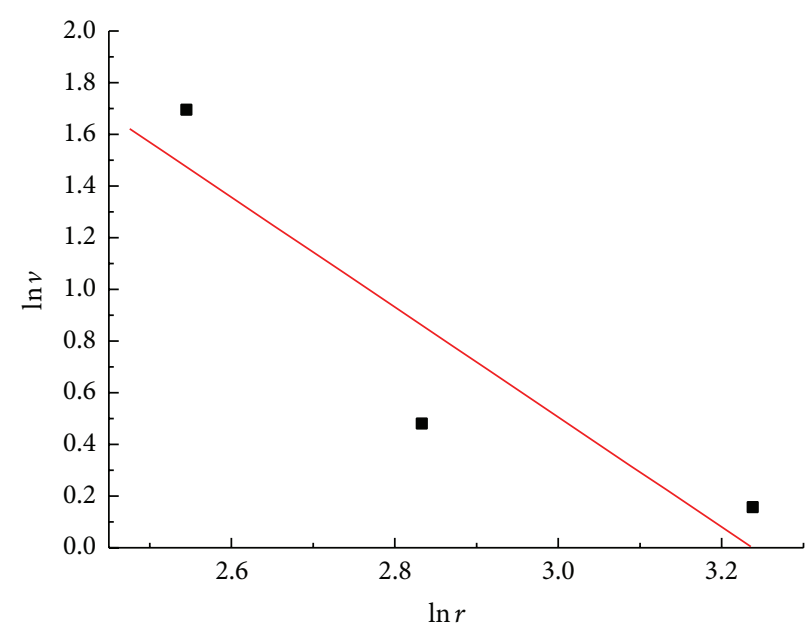

(c) Water-decoupling charging structure

FIGURE 4: Fitting curve of three charging structures. 
TABLE 2: Value of $k$ and $\alpha$ in fitting vibration formula of different charging structures.

\begin{tabular}{lcccc}
\hline Charging structure & $k$ & $\alpha$ & Correlation coefficient & $k$ 's value with $\alpha=1.3$ \\
\hline Coupling & 136.866 & 1.320 & -0.99986 & 131.368 \\
Air-decoupling & 28.479 & 0.872 & -0.99231 & 101.646 \\
Water-decoupling & 47.500 & 1.157 & -0.97652 & 71.586 \\
\hline
\end{tabular}

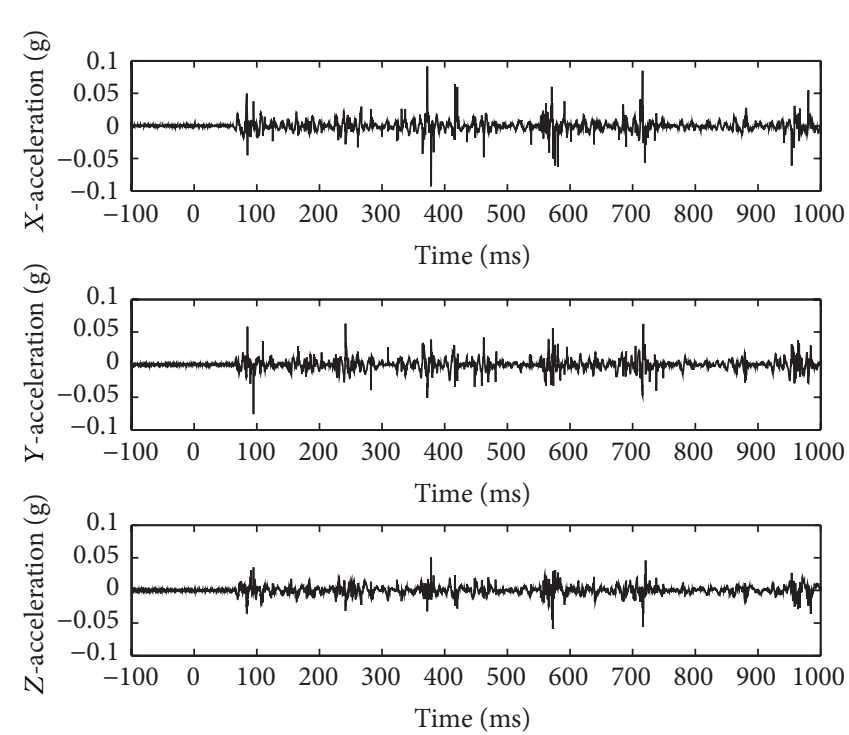

(a) Coupling charging structure
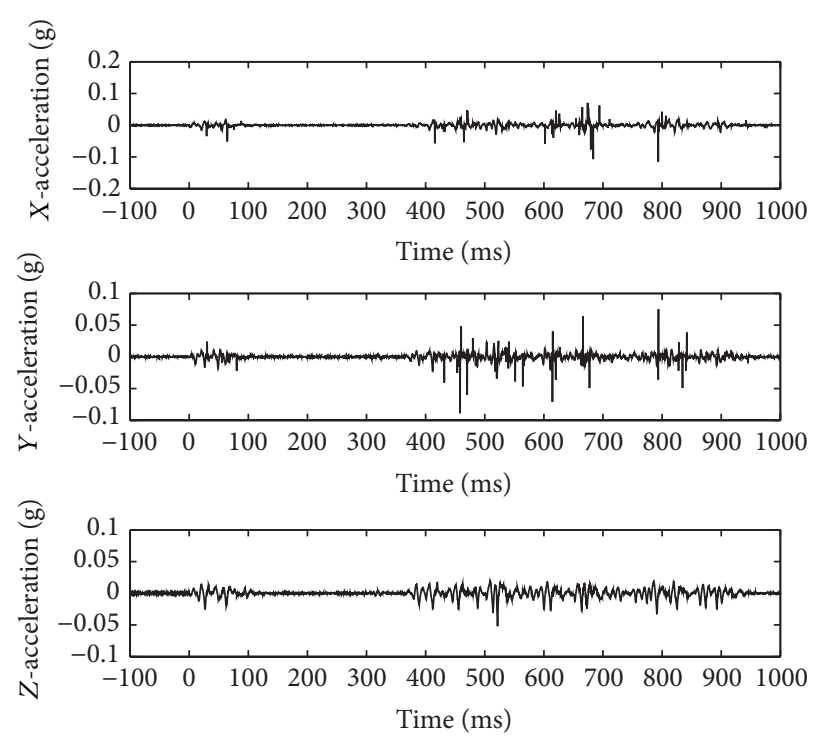

(b) Water-decoupling charging structure

FIGURE 5: Schedule chart of different charging structures' vibration acceleration in three directions.

determined, vibration velocity formula of three charging structures can be compared as follows:

$$
\begin{aligned}
v_{\text {coupling }} & =131.368 r^{-1.3}>v_{\text {air-decoupling }}=101.646 r^{-1.3} \\
& >v_{\text {water-decoupling }}=71.586 r^{-1.3} .
\end{aligned}
$$

In blasting rocks, instantaneous energy $E$ of blasting vibration at a moment is proportional to $v^{2}$; that is, instantaneous energy of blasting vibration is proportional to vibration velocity's quadratic [16]; hence, instantaneous energy of blasting vibration carried by seismic wave of measuring points can be reflected by vibration velocity. With formula (3), relationship between instantaneous energy of blasting vibration of three charging structures is

$$
E_{\text {coupling }}>E_{\text {air-decoupling }}>E_{\text {water-decoupling }} \text {. }
$$

At measuring points with close rational distance, that is, $r=12.842$ in coupling charging and $r=12.747$ in waterdecoupling charging, when charging structures are different, time-history curves of acceleration in three directions are shown in Figure 5, and peak value of vibration acceleration at that rational distance is about $0.1 \mathrm{~g}$. If there exist structural buildings near the blasting area, a series of protective actions should be taken $[17,18]$. Comparative relationship of vibration acceleration intensity of blasting with different charging structures is in accordance with conclusions in (3) on comparative relationship of vibration velocity intensity as well as (4) on comparative relationship of energy intensity.

\section{Theoretical Research}

Explosion produces detonation wave in the hole and when transmitting between different media, there may be refraction and reflection at the interface due to different wave impedance; thus, energy transmitted in wave may change. Test data suggest that, with different charging structures, detonation products have different effects on hole wall, distribution pressure produced on hole wall is different, and so is vibration energy at the measuring points of far region of blasting. According to speed fitting formula (3), vibration energy transmitted in coupling charging structure is the largest and that of water-decoupling charging is the smallest. From the perspective of impedance matching, when detonation products equal rock impedance, detonation products of coupling charging directly affect hole wall; explosive of air-decoupling charging structure explodes in the air, and detonation products inflate and fill the blasting hole and then affect hole wall; explosive of water-decoupling charging structure explodes in the water, and detonation products compress the medium water and then transmit blasting energy to rock. To sum up, when rock impedance is determined, detonation products of different charging structures have different impedance on the hole wall, so impedance matching relationships are different, suggesting that charging structure will have influence on blasting vibration energy.

No matter which kind of charging structure is adopted, detonation products will keep touching rock at the interface. 
And, according to continuous conditions and Newton's third law, after reflection and transmission, velocity and stress of the particle on interface's both sides are equal; therefore, the following equation group can be deduced $[3,19]$ :

$$
\begin{gathered}
v_{I}+v_{R}=v_{T}, \\
\sigma_{I}+\sigma_{R}=\sigma_{T} .
\end{gathered}
$$

Here, $v$ stands for particle's velocity, $\sigma$ is particle's stress, and subscripts $I, R$, and $T$, respectively, refer to relevant elements disturbed by incident, reflection, and transmission. As momentum of wave-front conserves

$$
\frac{\sigma_{I}}{\rho_{b} C_{b}}-\frac{\sigma_{R}}{\rho_{b} C_{b}}=\frac{\sigma_{T}}{\rho_{y} C_{y}},
$$

combine (5) (6) as follows:

$$
\begin{aligned}
\sigma_{R} & =F \sigma_{I}, \\
v_{R} & =-F v_{I}, \\
\sigma_{T} & =T \sigma_{I}, \\
v_{T} & =n T v_{I}, \\
n & =\frac{\left(\rho_{b} C_{b}\right)}{\left(\rho_{y} C_{y}\right)} .
\end{aligned}
$$

Here, $\rho_{b} C_{b}$ and $\rho_{y} C_{y}$, respectively, stand for denotation products of the explosive and rock's impedance, with $n$ as impedance ratio. Combine the equations, and then transmission coefficient $T=2 /(1+n)$ and reflection coefficient $F=$ $(1-n) /(1+n)$ can be calculated. Therefore, $T$ and $F$ depend on impedance ratio of denotation products and the rock. When $n=1, \rho_{b} C_{b}=\rho_{y} C_{y}, T=1$, and $F=0$, the largest amount of energy can be transmitted from denotation products to the rock.

3.1. Coupling Charging. Coupling charging means that explosive fills hole in direct contact with the hole wall. When explosive blows up, explosion products of denotation directly affect the hole wall, transmitting blasting energy to the rock to make it broken with cracks; then some energy can be transmitted in the form of seismic wave to far region of blasting. The transmission of blasting energy is relatively easy and according to the denotation theory of denotation explosive [13, 17], due to high denotation pressure, explosive's initial pressure can be negligible, and then denotation products' acoustic velocity and density can be

$$
\begin{aligned}
& D_{b}=\frac{\gamma}{\gamma+1} D_{z}, \\
& \rho_{b}=\frac{\gamma+1}{\gamma} \rho_{z} .
\end{aligned}
$$

Here, $\rho$ and $D$, respectively, are density and velocity, subscripts $z$ and $b$ stand for explosive and detonation products, and $\gamma$ stands for adiabatic index. When detonation products' wave impedance equals that of the explosive (i.e., impedance ratio $n=1$ ), the largest amount of energy from explosion can get transmitted into the rock. Though it is generally difficult to realize impedance matching, according to relevant theories, explosives with high impedance can better help transmit explosive energy to rocks with high impedance.

3.2. Air-Decoupling Charging. When air-decoupling charging structure was adopted, charging diameter $d_{z}$ is different from that of blasting hole $d_{0}$ and decoupling coefficient of charging is $k_{d}$ :

$$
k_{d}=\frac{d_{0}}{d_{z}} .
$$

After explosive blasting, detonation products firstly transmitted in air cushion, forming air shock wave and acting on the hole wall. Detonation products expand adiabatically in the hole. Ignore quality of the air and then relationship between decoupling coefficient and density of the denotation products $\rho_{k}$ is

$$
\frac{\rho_{b}}{\rho_{k}}=\frac{d_{0}^{2}}{d_{z}^{2}}=k_{d}^{2} .
$$

Subscript $k$ stands for correlative of detonation products in the air after explosion (same as below). Combine (9) (11):

$$
\rho_{k}=\frac{\gamma+1}{\gamma} k_{d}^{-2} \rho_{z} .
$$

Regardless of other factors' influence, detonation products in the hole follow adiabatic expansion; then

$$
\begin{aligned}
\frac{P_{k}}{P_{b}} & =\left(\frac{\rho_{k}}{\rho_{b}}\right)^{\gamma}, \\
P_{b} & =\frac{1}{8} \rho_{z} D_{z}^{2} .
\end{aligned}
$$

Here, $P_{k}$ refers to detonation products' pressure that fills the hole and $P_{b}$ is detonation products' initial pressure upon explosive's blasting. Combine (13):

$$
P_{k}=\frac{1}{8} k_{d}^{-2 \gamma} \rho_{z} D_{z}^{2} .
$$

Relationship between detonation products' acoustic velocity $D_{k}$ and pressure in the hole $P_{k}$ is

$$
D_{k}=\left(\frac{\gamma P_{k}}{\rho_{k}}\right)^{1 / 2}=\frac{\sqrt{2(\gamma+1)}}{4(\gamma+1)} \gamma k_{d}^{1-\gamma} D_{z} .
$$

Combine (12) and (15); it can be seen that, in air-decoupling charging structure, impedance of detonation products operating on the wall of borehole after explosion is

$$
\rho_{k} D_{k}=\frac{\sqrt{2(\gamma+1)}}{4} k_{d}^{-\gamma-1} \rho_{z} D_{z} .
$$


Supposing adiabatic exponent $\gamma$ is determined, in airdecoupling charging structure, relationship between detonation products' impedance and explosive's impedance is relevant to decoupling coefficient. According to impedance matching theory, when impedance of detonation products equals that of the rock, energy produced by explosion can be transmitted to the rock more effectively; that is

$$
\rho_{k} D_{k}=\frac{\sqrt{2(\gamma+1)}}{4} k_{d}^{-\gamma-1} \rho_{z} D_{z}=\rho_{y} D_{y} .
$$

As decoupling coefficient $k_{d}$ is larger than $1, \rho_{k} D_{k}$ decreases with the increase of $k_{d}$. From the perspective of impedance matching, to better transmit blasting energy, it is necessary to synthetically analyze relationship between the rock's impedance of blasting area, explosive's categories, and decoupling coefficient of the charging. When rock and explosive are determined, if air-decoupling charging structure is selected, decoupling coefficient in (17) should be adopted to advance the transmission of blasting energy.

3.3. Water-Decoupling Charging. When adopting waterdecoupling charging structure to blast, detonation products expand and compress the water, resulting in changes of water's density and wave velocity. Based on adiabatic expansion rule and the theory of fluid mechanics [20], the following equations can be concluded:

$$
\begin{aligned}
\frac{\rho_{s}}{\rho_{s 0}} & =\frac{d_{0}^{2}-d_{z}^{2}}{d_{0}^{2}-\left(d_{z}+\omega\right)^{2}} \\
D_{s} & =\left(\frac{k_{w}}{\rho_{s}}\right)^{1 / 2} \\
d P & =\frac{k_{w}}{V} d V .
\end{aligned}
$$

Here, $\rho_{s}$ is water's density under the influence of the detonation, $\rho_{s 0}$ is water's original density, $\omega$ refers to water's radial compression in the expanding and compressing process, $D_{s}$ stands for water's acoustic velocity after compression, and $k_{w}$ stands for water's bulk modulus; $P$ and $V$, respectively, refer to pressure and water's volume in the compressing process.

Combine (18) and reorganize it to get

$$
\rho_{s}=\rho_{s 0} e^{\left(\rho_{z} D^{2} / 8 k_{w}\right)\left(d_{z} /\left(d_{z}+\omega\right)\right)^{6}} .
$$

Combine (18) (19) and then water's impedance after detonation products' compression is

$$
\rho_{s} D_{s}=\left[k_{w} \rho_{s 0} e^{\left(\rho_{z} D^{2} / 8 k_{w}\right)\left(d_{z} /\left(d_{z}+\omega\right)\right)^{6}}\right]^{1 / 2} .
$$

Equation (20) shows that impedance matching relationship of water charging is different from that of coupling structure or air-decoupling charging structure. With water-decoupling charging structure, relationship between impedance detonation products acting on the hole wall and explosive's impedance turns into exponential relationship.
To realize impedance matching between detonation products and the rock, not only charging radius but also bulk modulus of the water should be taken into consideration. Consequently, impedance matching can be achieved through adjusting the decoupling coefficient. And when rock and explosive are determined, explosive's utilization can be further improved with adjustment on decoupling coefficient.

With analysis of explosion energy's transmission of three charging structures from the perspective of impedance matching [21, 22], it can be summarized that impedance of explosive and rock directly influences explosive energy's transmission. No matter which charging structure to take, the rock with high impedance requires explosive with high impedance to better utilize explosion energy. When both explosive and rock are determined, different decoupling media result in different influences of decoupling coefficients; nevertheless, formulas can be employed to calculate and get a decoupling coefficient realizing impedance matching of detonation products and the rock.

\section{Discussions}

Intensity of shock wave of explosion inside the rock quickly decreases with the increase of transmitting distance. According to damage characteristics of rock blasting, with explosion as the center, there are crushed zone, fracture zone, and vibration zone [15], from the blasting center to distant area. Analysis on test data suggests that charging structure has a significant influence on blasting vibration energy. Under the geological condition of present test, comparison of three charging structures confirms that when proportional distance of the measuring point is determined, coupling charging generates blasting larger vibration energy, blasting vibration energy of decoupling charging is relatively small, and blasting vibration energy of water-decoupling charging is smaller than that of air-decoupling charging. The present paper identified influence of different charging structures on explosion energy's transmission from the perspective of impedance matching, suggesting that advanced charging structure can better transmit explosion energy. Combining test and theory, charging structure apparently plays a significant role in transmission and distribution of blasting energy; thus, adjusting charging structure in blasting excavation engineering can help enlarge rock's fracture zone and reduce explosion energy's distribution in crushed zone and vibration zone.

Explosive of coupling charging structure directly operates on the hole wall; hence the instant the explosive blasts (regarding the explosive as completely detonating) detonation products operate on hole wall and directly transmit explosion energy to rock. On the basis of theoretical analysis in Section 3, when impedance of explosive matches that of rock, explosion energy can be completely transmitted to rock and a large amount of explosion energy is consumed around the explosive for rock smashing. Energy consumption occurs before shock wave as well as behind wave-front; part of the energy is transformed into left elastic deformation energy and another part of the energy is sent out in the form of elastic wave (seismic wave) [23]. According to (4), when analyzed 


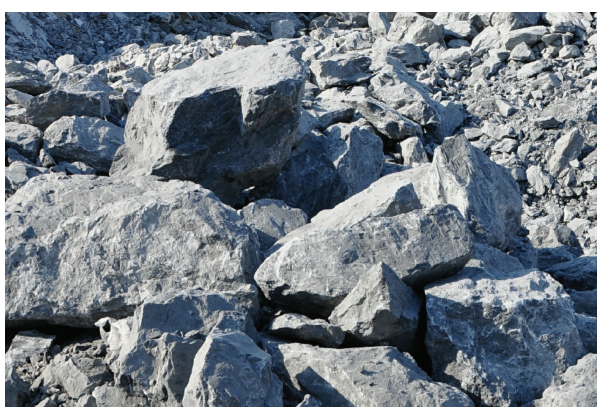

(a) Coupling charging structure

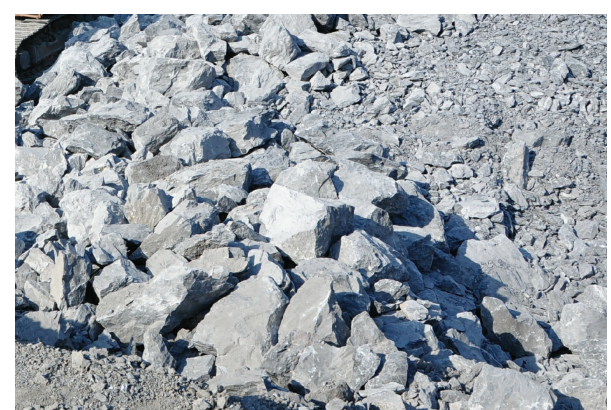

(b) Air-decoupling charging structure

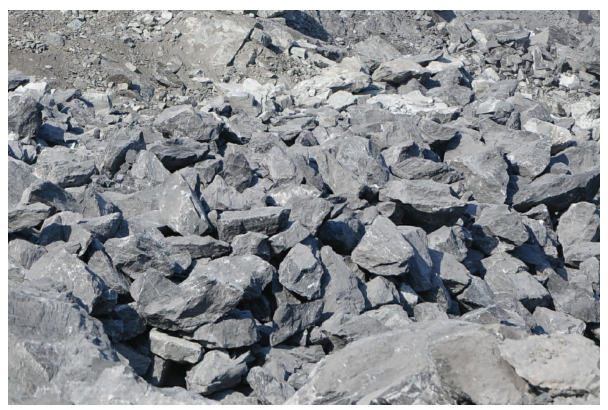

(c) Water-decoupling charging structure

Figure 6: The effect diagram of blasting of different charge structures.

energy is carried by seismic wave, coupling charging generates the largest amount of blasting vibration energy; in other words, energy is transformed into elastic wave to the largest extent, but the energy is unavailable and increases damaging effects of blasting vibration. Thus, the less ratio energy accounts for, the more effective utilization of explosive can be achieved. In addition, when coupling charging explodes, explosion energy acts on hole wall and then the intensity shock wave generated is far more than rock's dynamic compressive strength. As produced explosion energy will enlarge rock's crushed area, more explosion energy can be applied to rock smashing. Besides, blasting rocks requires appropriate rock size for convenient transportation and reuse; therefore, to reduce explosion energy on smashing rocks and enlarge rock's fracture zone, better charging structure is essential for the engineering.

When decoupling charging structure is adopted, detonation products firstly operate on decoupling medium and then transmit explosive energy to the hole wall. When air is selected as decoupling medium, explosive explodes in the air and detonation products act on hole wall after filling the hole. According to impedance matching theory, when explosive and rock are determined, decoupling coefficient decides impedance relationship between detonation products operating on hole wall and the rock. Impedance of explosive adopted in the test is smaller than that of the rock, so, based on (17), with the increase of decoupling coefficient, ratio of detonation energy transmitted to rock decreases. And it can also get demonstrated for the test suggests that, at measuring points with the same proportional distance, explosion vibration energy generated by air-decoupling charging is smaller than that of coupling charging.
Water and air have different physical properties; thus, water has more apparent incompressibility, while water is compressible for rock. When explosion's detonation products are transmitted in water, energy of detonation shock wave may decrease; however, detonation energy acting on hole wall can be more evenly distributed.

Test data confirm that, at measuring points with the same proportional distance, explosion vibration velocity generated by water-decoupling charging structure is the smallest in three charging structures. Equation (20) is based on theoretical derivation, stating that impedance relationship between detonation products produced by water-decoupling charging and rock is relatively complex and it is not only related to decoupling coefficient, but also influenced by water's radial compression in the hole. At the test scene, when waterdecoupling charging structure is adopted, rock dust is obviously produced rarely in blasting area, which reveals blasting dust can be effectively reduced by water-decoupling charging structure.

Through observation on rock fragmentation after blasting and analysis of pictures (like Figure 6), whether from the perspective of impedance matching theory or actual situations in tests, it turns out that charging structures have influence on blasting effects, especially water-decoupling charging for it can enhance the utilization of explosive energy and distribution of explosion rock fragmentation. According to tests and theoretical analysis, when explosive and rock are fixed, the closer their impedance matching is, the more the explosion energy can be transmitted to the rock. And, for coupling charging structures, without transmission through decoupling media, explosion energy cannot be evenly operated on rocks; thus, crushing zone's scope will be larger than that 
of decoupling charging structure. With different decoupling media, impedance matching relationships between explosives and rocks may change. And, in tests, when used as decoupling medium, water can help operate explosion energy on rocks and can generate the least crushing zone in blasting.

Hence, decoupling charging is beneficial to utilization of explosive crushing [24, 25]. When coupling charging structure is adopted, operating time of explosive's blasting is short and its instantaneous energy carried when transmitting to the rock is larger than that of decoupling charging, so more energy is consumed on rock smashing. Consequently, decoupling charging can effectively reduce the initial shock pressure acting on hole wall and prolong operating time to make detonation energy slowly act on the rock; therefore, instantaneous energy can be reduced, and it can be ensured to use more detonation energy on enlarging fracture zone and reduce detonation energy in crushed zone and vibration zone. In summary, vibration velocity of coupling charging structure in far region of blasting is larger than that of decoupling charging.

\section{Conclusions}

With test and theoretical analysis, this research presented the influence of charging structure on blasting vibrating energy and the following conclusions can be obtained:

(1) Charging structure greatly influences instantaneous energy of blasting vibration and blasting effects: decoupling charging structure can prolong detonation products' operating time, distribute explosion energy more evenly, reduce instantaneous energy of blasting vibration, and apply more explosions to fracture zone. Furthermore, water-decoupling charging structure has the best effects on reducing vibration and generating the most even rock fragmentation.

(2) Analyzed from the perspective of impedance matching, impedance of explosive and rock has a pivotal impact on transmission of explosion energy: explosive with high impedance can help better transmit energy of high-impedance rock blasting. No matter which charging structure to choose, matching relationship determines energy projection; therefore, different engineering requirements need comprehensive analysis and selection of appropriate charging structures.

(3) Decoupling charging structure's medium and coefficient can influence blasting vibration energy and effects to some extent. For different engineering requirements, different charging structures should be adopted and appropriate decoupling coefficient should be selected to help improve explosion utilization and achieve better blasting effects.

\section{Conflict of Interests}

The authors declare that there is no conflict of interests regarding the publication of this paper.

\section{Acknowledgments}

This work was supported by a grant from Department of Infrastructure Barracks, Grant no. KYGYZXJK0914. Their contribution to bringing this project to fruition is much appreciated. In addition, the authors would like to express their gratitude to Blasting Technology Service Department of College of Field Engineering for their invaluable support and considerable help. Meanwhile, sincere thanks go to graduated students Wang Zheng, Qin Ruping, and Chen Xueping of the laboratory, who have offered selfless devotion and profound concern in completion of the research. And high tribute will be paid to Liang Ting, who has provided insightful comment on editing and translating for this thesis. The authors would like to express their appreciation to the referees for making valuable advice in contributing to this paper.

\section{References}

[1] J. R. Brickman, "Crushing mechanism separation shock wave and gas expansion effects," in Proceedings of the 2nd International Symposium on Rock Blasting, pp. 1-8, Yangtze River Scientific Research, Wuhan, China, 1990.

[2] X. Xie, Precision Blasting, Huazhong University of Science and Technology Press, Wuhan, China, 2010.

[3] J. Ning, C. Wang, and T. Ma, Explosion and Shock Dynamics, National Defense Industry Press, Beijing, China, 2010.

[4] P. R. Day, "Controlled blasting to minimize over break with big boreholes underground," in Proceedings of the 8th Conference on Explosives and Blasting Techniques, pp. 262-274, Louisiana State University, New Orleans, La, USA, January-February 1982.

[5] Q. Zong and D. Meng, "Influence of different kinds of hole charging structure on explosive energy transmission," Chinese Journal of Rock Mechanics and Engineering, vol. 22, no. 4, pp. 641-645, 2003.

[6] J. Chen, C. Bai, Y. Zeng, Z. Wang, and J. Li, "Experiment study of charging constriction influence on explosion seismic effect," in Proceedings of the National Security Emergency and Hazardous Substances Technical Symposium, pp. 1098-1103, 2013.

[7] Z.-L. Wang and Y.-C. Li, "Numerical simulation on effects of radial water-decoupling coefficient in engineering blast," Rock and Soil Mechanics, vol. 26, no. 12, pp. 1926-1930, 2005.

[8] R. Pape, K. R. Mniszewski, and A. Longinow, "Explosion phenomena and effects of explosionson structures. I-III," Practice Periodical on Structural Design and Construction, vol. 15, no. 2, pp. 135-169, 2010.

[9] A. Longinow and K. R. Mniszewski, "Protecting buildings against vehicle bomb attacks," Practice Periodical on Structural Design and Construction, vol. 1, no. 1, pp. 51-54, 1996.

[10] W. M. Yan and K.-V. Yuen, "On the proper estimation of the confidence interval for the design formula of blast-induced vibrations with site records," Rock Mechanics and Rock Engineering, vol. 48, no. 1, pp. 361-374, 2015.

[11] P. Segarra, J. A. Sanchidrián, R. Castedo, L. M. López, and I. del Castillo, "Performance of some coupling methods for blast vibration monitoring," Journal of Applied Geophysics, vol. 112, pp. 129-135, 2015.

[12] Y. Nianhua, Theroy of Blasting Vibration and Testing Technology, China Railway Publishing House, Beijing, China, 2014.

[13] C. H. Dowing, Blast Vibration Monitoring and Control, vol. 3, Prentice Hall, Englewood Cliffs, NJ, USA, 1985. 
[14] L. P. Orlenko, Explosion Physics, Science Press, Beijing, China, 2011.

[15] D. Zhang, MATLAB Numerical Analysis, China Machine Press, Beijing, China, 2012.

[16] H. Li, W. Lu, D. Shu, X. Yang, and C. Yi, "Study of energy attenuation law of blast-induced seismic wave," Chinese Journal of Rock Mechanics and Engineering, vol. 29, supplement 1, pp. 3364-3369, 2010.

[17] A. Longinow, "Blast protection of buildings," Practice Periodical on Structural Design and Construction, vol. 18, no. 4, pp. 194-195, 2013.

[18] G. F. Revey, "Managing rock blasting work in urban environments," Practice Periodical on Structural Design and Construction, vol. 11, no. 2, pp. 86-92, 2006.

[19] L. Wang, Foundation of Stress Waves, National Defense Industry Press, Beijing, China, 2005.

[20] J. Ye, Z. Liu, and S. Xu, Fluid Mechanics, Fudan University Press, Shanghai, China, 1989.

[21] Q. Zhang, "Explosion and impedance matching between explosive and rock," Coal Science and Technology, vol. 18, no. 7, 1990.

[22] X. Li, "The energy study of impedance matching between rock and explosive," Journal of Mid-South Mining Industry Academy, vol. 23, no. 1, 1992.

[23] Q. Qian and M. Wang, Impact and Explosion Effects in Rock and Soil, National Defense Industry Press, Beijing, China, 2010.

[24] W. Liang, H. Liu, and F. Zhou, "Influence of air-decoupling charge on rock blasting," Transactions of Beijing Institute of Technology, vol. 32, no. 12, pp. 1215-1218, 2012.

[25] W. Wang, X.-C. Li, L. Shi, and Z.-M. Fang, "Discussion on decoupled charge loosening blasting in deep rock mass," Rock and Soil Mechanics, vol. 29, no. 10, pp. 2837-2842, 2008. 

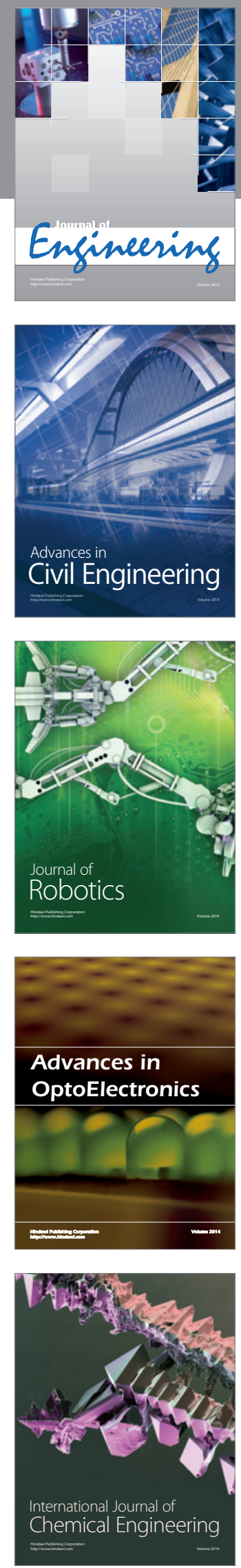

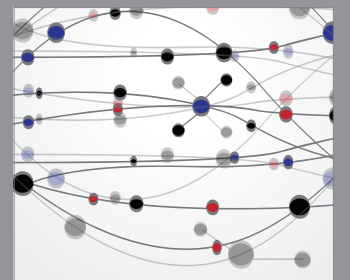

The Scientific World Journal
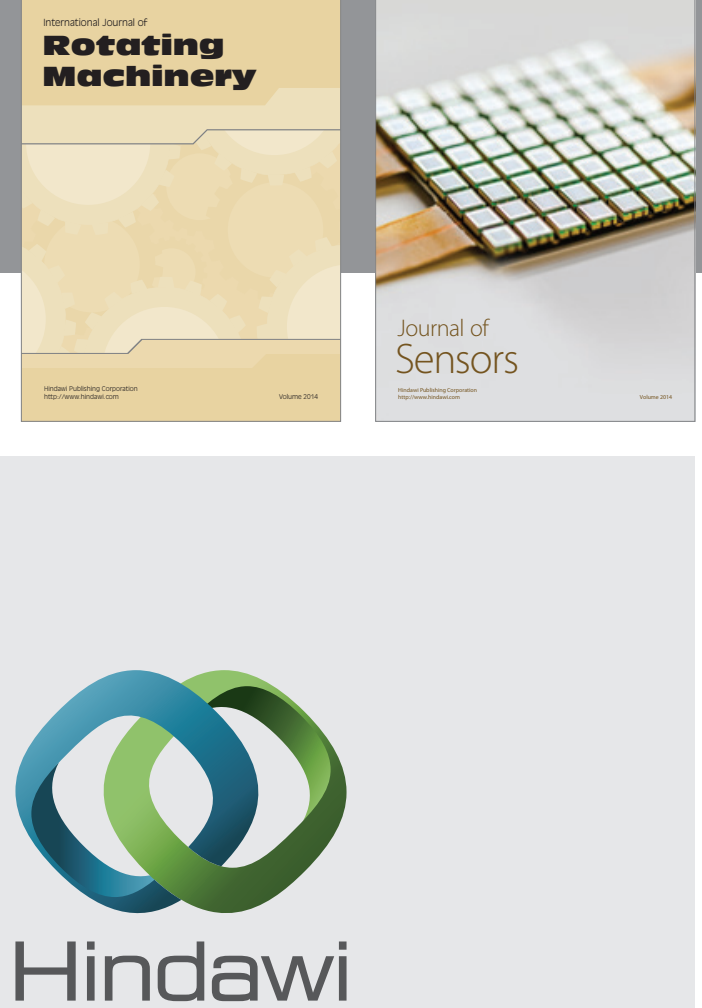

Submit your manuscripts at http://www.hindawi.com
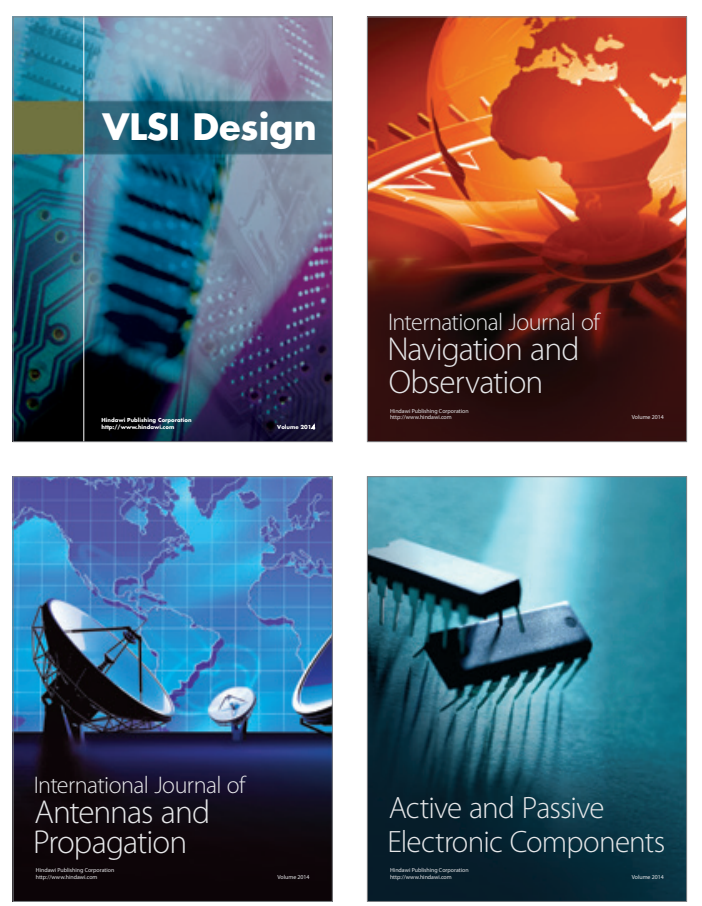
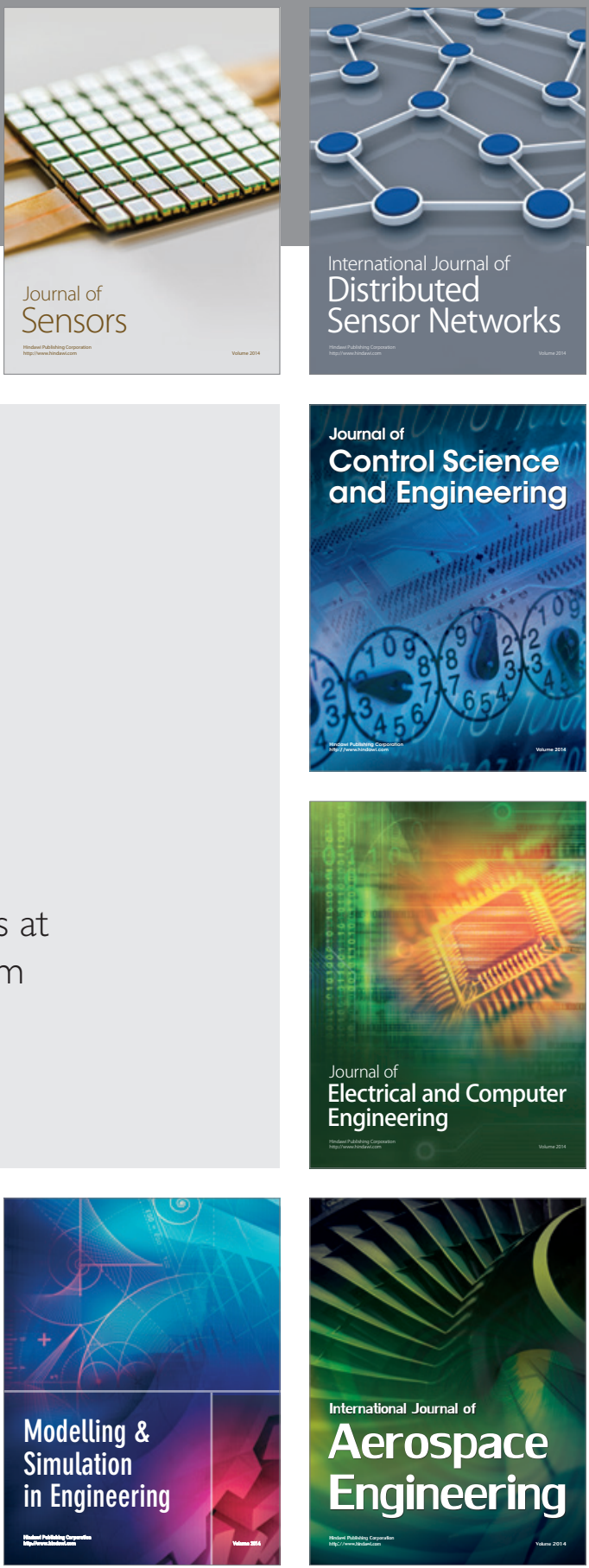

Journal of

Control Science

and Engineering
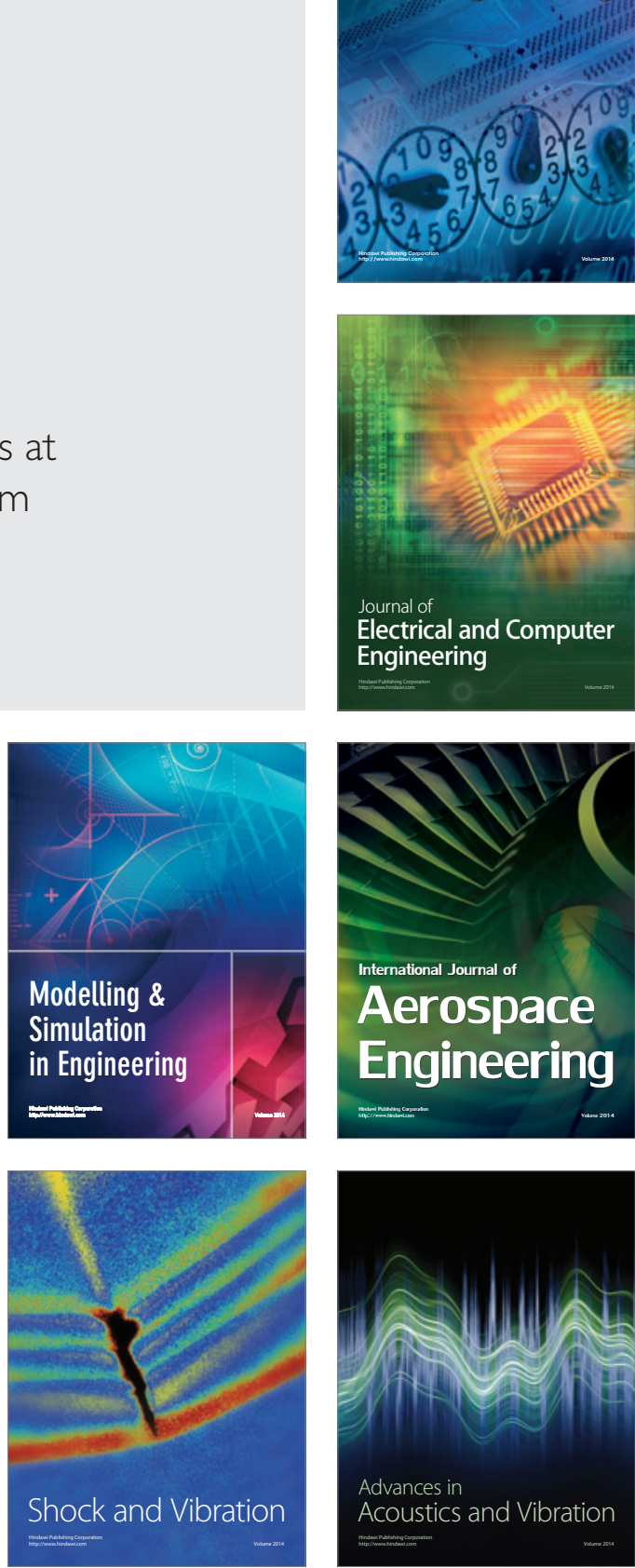